\title{
Global Periodic Attractors for a Class of Infinite Dimensional Dissipative Dynamical Systems
}

\author{
Hongyan Li \\ College of Management, Shanghai University of Engineering Science, Shanghai, China \\ Email: lihongyan580@sohu.com
}

Received May 2, 2013; revised June 10, 2013; accepted July 5, 2013

Copyright (C) 2013 Hongyan Li. This is an open access article distributed under the Creative Commons Attribution License, which permits unrestricted use, distribution, and reproduction in any medium, provided the original work is properly cited.

\begin{abstract}
In this paper we consider the existence of a global periodic attractor for a class of infinite dimensional dissipative equations under homogeneous Dirichlet boundary conditions. It is proved that in a certain parameter, for an arbitrary timeperiodic driving force, the system has a unique periodic solution attracting any bounded set exponentially in the phase space, which implies that the system behaves exactly as a one-dimensional system. We mention, in particular, that the obtained result can be used to prove the existence of the global periodic attractor for abstract parabolic problems.
\end{abstract}

Keywords: Dissipative Equation; Global Periodic Attractor; One-Dimensional System

\section{Introduction}

In this paper, we consider a class of infinite dimensional dissipative equations with the Dirichlet boundary condition

$$
\left\{\begin{array}{l}
u_{t}+A(t) u+g(u)=f(x, t), \quad(x, t) \in \Omega \times R_{+}, \\
\left.u(x, t)\right|_{\partial \Omega}=0, \quad t \geq 0 \\
u(x, 0)=u_{0}(x), \quad x \in \Omega
\end{array}\right.
$$

where $u=u(x, t)$ is a real-valued function on $\Omega \times[0,+\infty), \Omega$ is an open bounded set of $R^{n}(n \in N)$ with a smooth boundary $\partial \Omega, u(x, t)$ takes values in a Hilbert space $H$, the family $\{A(t): t \in[0, \infty)\}$ of unbounded linear operators generates a linear evolution operator. The external force term $f(., t)$ is continuous and $\omega$-periodic function in $t$, where $\omega$ is a positive constant. Let $g \in C^{1}(R, R)$. There exists a nonnegative constant $c_{1} \geq 0$, such that

$$
\left|g\left(u_{1}\right)-g\left(u_{2}\right)\right| \leq c_{1}\left|u_{1}-u_{2}\right|, \quad \forall u_{1}, u_{2} \in R,
$$

where $|$.$| denotes the absolute value of the number in$ $\mathrm{R}$.

There has been an increasing interest in the study of the evolution equations of form (1.1), such as existence and asymptotic behavior of solutions (mild solutions, strong solutions and classical solutions), and existence of global attractors, etc. Especially in physics and mechanics, many important results associated with this problem have been obtained in [1-7]. In [9] and [10], Hernandez and Henriquez have extended the problem studied in [8] to neutral equations and established the corresponding existence results of solutions and periodic solutions. In their work, $A(t)=A$, especially, $A=-\Delta$ is a negative Laplacian operator, and A generates an analytic semigroup so that the theory of the fractional power has been used effectively there. However, their results clearly cannot apply to Equation (1.1) with $A(t)$ is non-autonomous which is a more general and maybe more important case [11]. So we will use the appropriate assumptions to overcome the difficulty for the non-autonomous operator $A(t)$.

We arrange this paper as follows. Firstly we present the existence and uniqueness of solutions. Then we obtain a nonstandard estimation under which system (1.1) possesses a global periodic attractor. Finally, for the special case $A(t)=-\Delta$, we discuss the existence of a global periodic attractor for abstract parabolic problems.

\section{Preliminaries}

For the family $\{A(t): t \in[0, \infty)\}$ of linear operators, we impose on the following restrictions:

1) The domain $D(A)$ of $\{A(t): t \in[0, \infty)\}$ is dense in Hilbert space $H$ and independent of $t, A(t)$ is a closed linear operator;

2) For each $t \in[0, \infty)$, the resolvent $R(\lambda, A(t))$ exists for all $\lambda$, with $\operatorname{Re} \lambda \leq 0$ and there exists $C_{0}>0$ 
so that $\|R(\lambda, A(t))\| \leq C_{0} /(|\lambda|+1)$;

3) There exists $0<\alpha \leq 1$ and $C_{1}>0$ such that

$$
\left\|(A(t)-A(s)) A^{-1}(\tau)\right\| \leq C_{1}|t-s|^{\alpha}
$$

for all $t, s, \tau \in[0, \infty)$;

4) For each $t \in[0, \infty)$ and some $\lambda \in \rho(A(t))$, the resolvent set of $A(t)$, the resolvent $R(\lambda, A(t))$, is a compact operator.

Then the family $\{A(t): t \in[0, \infty)\}$ generates a unique linear evolution operator $U(t, s), 0 \leq s \leq t<\infty$, satisfying the following properties:

1) $U(t, s) \in L(H)$, the space of bounded linear transformations on $H$, whenever $0 \leq s \leq t<\infty$ and for each $u \in H$, the mapping $(t, s) \rightarrow U(t, s) u$ is continuous;

2) $U(t, s) U(s, \tau)=U(t, \tau)$ for $0 \leq \tau \leq s \leq t<\infty$;

3) $U(t, t)=I$;

4) $U(t, s)$ is a compact operator whenever $t-s>0$;

5) $\frac{\partial}{\partial t} U(t, s)=-A(t) U(t, s)$, for $s<t$;

6) There is a constant $C_{2} \geq 1$ such that $\|U(t, s)\| \leq C_{2}, \quad 0 \leq s \leq t<\infty$;

7) If $0<h<1, t-s>h$, and $0<\gamma<1$, then

$$
\|U(t+h, s)-U(t, s)\| \leq \frac{c_{2}^{\prime} h^{\gamma}}{|t-s|^{\gamma}}
$$

for some $c_{2}^{\prime}>0$;

8) If $f(t)$ is continuous on $[0, \infty)$, then the function $t \rightarrow \int_{0}^{t} U(t, s) f(s) \mathrm{d} s$ is Holder continuous with any exponent $0<\gamma<1$.

Condition 4) ensures the generated evolution operator satisfies 4) (see [6], Proposition 2.1).

Proposition 1 (see [11]) The family of operators $\{U(t, s): t>s\}$ is continuous in $\mathrm{t}$ in the uniform operator topology uniformly for $s$.

Lemma 1 (see [11]) Consider the initial value problem (1.1) in E. If 1)-4) hold, then, for any $u_{0} \in H$, there exists a unique continuous function $u()=.u\left(., u_{0}\right) \in C\left(R_{+} ; H\right)$ such that $u\left(0, u_{0}\right)=u_{0}$ and $u(t)$ satisfies the integral equation

$$
u\left(t, u_{0}\right)=U(t, 0) u_{0}+\int_{0}^{t} u(t, s) f((x, s)-g(u)) \mathrm{d} s .
$$

$u(t)$ is called a mild solution of (1.1).

By Lemma 1 , the (mild) solution $u\left(t, u_{0}\right)$ of (1.1) determines a map $S(t)$ from $H$ into itself: $u_{0} \rightarrow u(t)$. Obviously, $\{S(n T) / n \in N\}$ is a discrete semidynamic system in $H$, since $f(x, t)$ is a $\omega$-periodic function with respect to $t \in R$.

\section{Main Result}

Theorem 1 Assume that (1.2), 1)-4) and $c_{1}\|u\|^{2}<\left\langle A(t)_{u, u}\right\rangle_{H}$ hold, then system (1.1) has a unique continuous $\omega$-periodic solution which attracts any bounded set exponentially. The process $\{S(t)\}_{t \geq 0}$ associated with (1.1) possesses a global periodic attractor.

Proof. Let $u_{1}(t), u_{2}(t)$ be two solutions of problem (1.1) with initial values $u_{01}, u_{02} \in H$, and $u(t)=u_{1}(t)-u_{2}(t)$. Then by (1.1), we find

$$
\frac{\mathrm{d} u(t)}{\mathrm{d} t}+A(t) u+g\left(u_{1}\right)-g\left(u_{2}\right)=0 .
$$

Taking the inner scalar product of each side of (3.1) with $u(t)$ in $H$, and we see that

$$
\frac{1}{2} \frac{\mathrm{d}}{\mathrm{d} t}\|u\|^{2}+\langle A(t) u, u\rangle_{H}+\left\langle g\left(u_{1}\right)-g\left(u_{2}\right), u\right\rangle_{H}=0 .
$$

For the third term on the left of (3.2), by (1.2), we have

$$
-\left\langle g\left(u_{1}\right)-g\left(u_{2}\right), u\right\rangle_{H} \leq c_{1}\|u\|^{2} .
$$

From (3.1)-(3.3), we find

$$
\frac{\mathrm{d}}{\mathrm{d} t}\|u\|^{2} \leq-2\langle A(t) u, u\rangle_{H}+2 c_{1}\|u\|^{2}
$$

and if

$$
\langle A(t) u, u\rangle_{H}>2 c_{1}\|u\|^{2},
$$

we might as well assume

$$
-2\langle A(t) u, u\rangle_{H}+2 c_{1}\|u\|^{2} \leq-2 \sigma\|u\|^{2},
$$

from the Gronwall's inequality, we have that

$$
\|u(t)\| \leq \mathrm{e}^{-\sigma t}\left\|u_{0}\right\|, \forall t \geq 0 .
$$

Now considering $\omega$-mapping

$$
S(\omega): H \rightarrow H, S(\omega) u_{0}=u\left(\omega, u_{0}\right),
$$

where $u\left(t, u_{0}\right)$ is the solution of (1.1), $u\left(0, u_{0}\right)=u_{0}$. From (3.4), $\forall u_{1}, u_{2} \in H$, we obtain

$$
\left\|S(\omega) u_{1}-S(\omega) u_{2}\right\| \leq \mathrm{e}^{-\sigma \omega}\left\|u_{1}-u_{2}\right\| .
$$

Thus $S(\omega)$ is a contraction mapping. By Banach's fixed point theorem, there exists a unique fixed point $u^{*}$ for $S(\omega)$ in $H$ such that $S(\omega) u^{*}=u^{*}$. At the same time, since $\{S(n \omega) / n \in N\}$ is a discrete semidynamic system in $H$, we can deduce

$$
S(n \omega) u^{*}=S(\omega)^{n} u^{*}, \forall n \in N,
$$

and

$$
u^{*}(t+\omega)=S(t) u^{*}(\omega)=S(t) u^{*}=u^{*}(t),
$$

where $u^{*}(t)$ is the solution passing $u^{*} \in H$. Thus $u^{*}(t)=u\left(t, u^{*}\right)$ is a $\omega$-periodic solution of system (1.1). By (3.4), $u^{*}(t)$ attracts any bounded set exponentially, 
which is a global periodic attractor of System (1.1). The proof is completed.

\section{Examples}

In this section, as an illustration of the main result in Section 3, we consider one example of System (1.1) and get the corresponding results. We consider an evolution equation (i.e., $A(t)=-\Delta$ in (1.1)) studied in [2,3]:

$$
\frac{\mathrm{d} u}{\mathrm{~d} t}-\Delta u+g(u)=f(x, t),
$$

and if the function $f$ is continuous $\omega$-periodic in $t$, we have the following theorem.

Theorem 2 System (4.1) possesses a global $\omega$-periodic attractor which attracts any bounded set exponentially, if $c_{1}<\lambda_{1}$ (where $\lambda_{1}$ is the first eigenvalue of operator $-\Delta$ that subjects to the homogeneous Dirichlet boundary condition).

\section{Acknowledgements}

This work is supported by the National Natural Science Foundation of China under Grant 11101265 and 61075115.

\section{REFERENCES}

[1] J. K. Cholewa and T. Dlokto, "Global Attractors in Abstract Parabolic Problem,” Cambridge University Press, Cambridge, 2000. doi:10.1017/CBO9780511526404

[2] J. K. Hale, “Asymptotic Behavior of Dissipative Systems,” $A M S, 1988$.

[3] R. Temam, "Infinite-Dimensional Dynamical Systems in Mechanics and Physics,” Springer-Verlag, New York, 1997.
[4] M. A. Hussain, "On a Nonlinear Integrodifferential Equation in Banach Space,” Indian Journal of Pure and Applied Mathematics, Vol. 19, 1988, pp. 516-529.

[5] S. M. Rankin III, "Existence and Asymptotic Behavior of a Functional Differential Equation in Banach Space," Journal of Mathematical Analysis and Applications, Vol. 88, No. 2, 1982, pp. 531-542. doi:10.1016/0022-247X(82)90211-6

[6] W. E. Fitzgibbon, "Semilinear Functional Equations in Banach Space,” Journal of Differential Equations, Vol. 29, No. 1, 1978, pp. 1-14. doi:10.1016/0022-0396(78)90037-2

[7] C. Zhong, C. Sun and M. Niu, “On the Existence of Global Attractor for a Class of Infinite Dimensional Dissipative Nonlinear Dynamical Systems," Chinese Annals of Mathematics, Vol. 26, No. 3, 2005, pp. 393-400. doi:10.1142/S0252959905000312

[8] H. R. Henriquez, "Periodic Solutions of Quasi-Linear Partial Functional Differential Equations with Unbounded Delay,” Funkcialaj Ekvacioj, Vol. 37, No. 2, 1994, pp. 329343.

[9] E. Hernandez and H. R. Henriquez, "Existence Results for Partial Neutral Functional Differential Equations with Unbounded Delay," Journal of Mathematical Analysis and Applications, Vol. 221, No. 2, 1998, pp. 452-475. doi:10.1006/jmaa.1997.5875

[10] E. Hernandez and H. R. Henriquez, "Existence of Periodic Solutions of Partial Neutral Functional Differential Equations with Unbounded Delay," Journal of Mathematical Analysis and Applications, Vol. 221, No. 2, 1998, pp. 499-522. doi:10.1006/jmaa.1997.5899

[11] X. Fu and X. Liu, "Existence of Periodic Solutions for Abstract Neutral Non-Autonomous Equations with Infinite Delay," Journal of Mathematical Analysis and Applications, Vol. 325, No. 1, 2007, pp. 249-267. doi:10.1016/j.jmaa.2006.01.048 